\title{
Possibile mixoma endoventricolare sinistro in paziente con TVP degli arti inferiori: considerazioni da un caso clinico
}

Possible left endoventricular myxoma in a patient with deep vein thrombosis of the lower limbs: a case report

Daniela Galimberti ${ }^{a, *}$, Alessandro Navazio ${ }^{b}$, Carlo Salvarani $^{c}$, Gianluigi Bajocchi ${ }^{c}$, Paolo Dolzani ${ }^{\mathrm{d}}$, Elisa Assirelli ${ }^{\mathrm{d}}$, Anna M. Casali ${ }^{\mathrm{a}}$, Maria C. Leone ${ }^{a}$, Attila M. Pizzini ${ }^{a}$, Mauro Silingardi ${ }^{a}$, Dimitriy Arioli ${ }^{a}$, Lorenzo Morini ${ }^{a}$, Davide Favali ${ }^{a}$, Ido Iori ${ }^{a}$

\author{
a UO Medicina Interna 1, Centro Emostasi e Trombosi-Stroke Unit (Direttore: dr. Ido lori), \\ Azienda Ospedaliera S. Maria Nuova, Reggio Emilia \\ ${ }^{\mathrm{b}}$ UO Cardiologia, Azienda Ospedaliera S. Maria Nuova, Reggio Emilia \\ ' UO Reumatologia, Azienda Ospedaliera S. Maria Nuova, Reggio Emilia \\ ${ }^{\mathrm{d}}$ UO Immunoreumatologia e Rigenerazione Tissutale, Istituto Ortopedico Rizzoli, Bologna
}

Ricevuto il 5 luglio 2010; accettato il 3 marzo 2011

disponibile online il 29 aprile 2011

\section{KEYWORDS}

Left ventricular myxoma;

Deep vein thrombosis;

Interleukin 6;

Inflammation.

\begin{abstract}
Summary
Introduction: We describe an unusual case of left ventricular myxoma associated with deep vein thrombosis (DVT).

Materials and methods: A 79-year-old woman was admitted with bilateral proximal DVT that developed while she was on warfarin. The anticoagulant therapy had been started (after 2 weeks of low-molecular weight heparin) 1 month earlier in a Cardiologic Unit, where the patient had been treated for "left ventricular thrombosis associated with acute pericarditis". After discharge, the patient continued to experience malaise, recurrent dizziness, dyspnea, flushing, and progressively severe leg swelling despite transthoracic echocardiography (TTE) findings of normal left ventricular function. She was finally admitted to our Unit for ultrasound studies for possible DVT. On admission the INR was in the therapeutic range with increased C-reactive protein (CRP), thrombocytopenia, mild anemia, anti-PF4 antibodies, lupus anticoagulant (LAC),
\end{abstract}

\footnotetext{
* Corrispondenza: UO Medicina Interna 1, Azienda Ospedaliera S. Maria Nuova, v.le Risorgimento 57 - 42100 Reggio Emilia.

E-mail: galimberti.daniela@asmn.re.it (D. Galimberti).
} 
a positive direct Coombs test, and mildly increased anti-platelet and anticardiolipin antibody levels. Malignancy, SLE, and inherited thrombophilia were excluded by a broad-spectrum instrumental and laboratory investigation. Since heparin-induced-thrombocytopenia could not be excluded, the warfarin was replaced with fondaparinux $(7.5 \mathrm{mg} /$ day). Given the persistence on TTE of a "small floating mass" at the left ventricular apex, we suspected the previously diagnosed thrombosis was actually an unusually located myxoma, and this diagnosis was confirmed by CT and MRI. The patient also had markedly increased serum levels of interleukin 6 (IL-6), regarded by many as a marker of cardiac myxoma and the source of the constitutional symptoms and immunologic features ("activity") of these lesions. The patient is now well except for recurrent flushing episodes. The IL-6 level is still high although the ESR, CRP, LAC have normalized. Since isolated IL-6 increases are associated with recurrence in PMR patients, we consider our patient at risk for relapse of systemic illness.

Results: Three cases of cardiac myxoma associated with DVT have been reported. Myxoma production of IL-6 could explain the extracardiac symptoms and the inflammatory/autoimmune activation that caused the DVT.

Discussion: Although histologic confirmation is lacking, the features of this case strongly suggest an atypical-site myxoma, in particular the concordant findings generated by the various diagnostic exams and unchanging nature of the ventricular lesion during follow-up.

(c) 2011 Published by Elsevier Srl.

\section{Introduzione}

I mixomi, pur essendo una patologia di rara osservazione $(0,5$ casi/1.000.000/anno), sono i più frequenti tumori cardiaci primitivi benigni [1,2]; hanno origine mesenchimale [3] e localizzazione prevalente in atrio sinistro [4,5]; soltanto il $16 \%$ è localizzato in atrio destro [6] e il $4 \%$ a livello ventricolare [7]. Colpiscono in prevalenza il sesso femminile, l'età giovanile (30-60 anni) e nel $5 \%$ dei casi sono multipli; più spesso sporadici, possono essere familiari e/o associati a mixomi cutanei, mammari, adenomi surrenalici e ipofisari [8,9] (mixomi "complessi"). Possono essere asintomatici o causare:

- sintomi ostruttivi cardiaci, correlati a dimensioni e localizzazione [10];

- embolie, per frammentazione, più frequenti a livello cerebrale per la prevalente localizzazione in atrio sinistro [11];

- sintomi sistemici, come dispnea, dolore toracico, flushing, sudorazione, presincope, febbre, alterazioni flogistiche e immunitarie [12-14].

Sono inoltre descritti rarissimi casi associati a tromboembolismo venoso $[15,16]$.

Il caso clinico presentato mostra un'interessante e rara associazione tra mixoma e trombosi venosa profonda, oltre a un'importante attivazione flogistico-immunitaria sistemica e a valori abnormemente elevati di interleuchina 6 (IL-6).

Varie segnalazioni in letteratura evidenziano un incremento dei mediatori di flogosi nei portatori di mixoma e, in particolare, di IL-6 $[17,18]$, citochina polifunzionale a livello dei meccanismi bioumorali che collegano flogosi, sistema immunitario e trombosi $[19,20]$, considerata marker bioumorale di mixoma cardiaco "attivo" [21] e responsabile dei sintomi sistemici associati [22,23].

\section{Caso clinico}

Descriviamo il caso di una signora di 79 anni, ricoverata presso l'Azienda Ospedaliera S. Maria Nuova di Reggio Emilia nel settembre 2009 per trombosi venosa profonda (TVP) bilaterale degli arti inferiori in corso di terapia anticoagulante orale in range. In anamnesi isterectomia, successiva radioterapia per neoplasia 10 anni prima e ipertensione arteriosa controllata farmacologicamente. La paziente era stata dimessa dalla UO di Cardiologia da un mese con diagnosi di "pericardite acuta e formazione trombotica peduncolata ventricolare sinistra, diabete mellito di prima diagnosi, ipotiroidismo" e da allora era in trattamento con warfarin, acido acetilsalicilico (ASA), insulina e levotiroxina; l'enoxaparina era stata sospesa a distanza di 12 giorni dalla dimissione. Al domicilio tuttavia persistevano malessere, astenia, episodi presincopali associati a sudorazione e flushing e dopo circa una settimana comparivano dolore e edema ingravescenti agli arti inferiori. Controlli ecocardiografici transtoracici (TTE) eseguiti alla dimissione e dopo circa una settimana mostravano progressiva riduzione del versamento pericardico e persistenza della "formazione trombotica" apicale con normali dimensioni e cinetica del ventricolo sinistro. La paziente, infine, veniva sottoposta a ecocolor-Doppler venoso degli arti inferiori che mostrava TVP femoro-popliteo-distale a destra e popliteo-distale a sinistra, e ricoverata presso la UO Medicina Interna 1, Centro Emostasi e TrombosiStroke Unit.

All'ingresso era pallida e sofferente, con edemi e arrossamento agli arti inferiori. Gli esami di laboratorio in urgenza mostravano incremento di PCR $(7,66 \mathrm{mg} / \mathrm{dL})$ e D-dimero ( $5739 \mathrm{ng} / \mathrm{ml}$ ), normali valori di fibrinogeno e antitrombina III. Il numero delle piastrine, un mese prima nella norma $(245.000 / \mathrm{mmc})$, era ora ridotto $\left(89.000 / \mathrm{mm}^{3}\right)$, l'INR era in range $(2,29)$; coesisteva una modesta anemia (emoglobina $11,3 \mathrm{~g} / \mathrm{dL}$ ) normocromica normocitica. All'elettrocardiogramma si evidenziava persistente ritmo sinusale.

La comparsa di TVP prossimale bilaterale in corso di terapia anticoagulante orale poteva indicare una possibile ripresa di malattia neoplastica, mentre il calo della conta piastrinica suggeriva una trombocitopenia da eparina (HIT) innescata dal precedente trattamento con enoxaparina. Entrambe le ipotesi diagnostiche imponevano la sospensione del warfarin e il trattamento con fondaparinux, che veniva intrapreso al dosaggio di 7,5 mg/die. Nei giorni successivi gli 
esami strumentali (TAC toraco-addominale, ecografia tiroidea) e di laboratorio (marker neoplastici, screening trombofilico, ANA-test, anti-DNA) escludevano una sottostante neoplasia, una trombofilia ereditaria o una patologia autoimmune; emergeva tuttavia un'importante attivazione degli indici di flogosi aspecifici (VES, alfa 2 -globuline, PCR) e autoimmunitari, con positività del lupus anticoagulante (LAC), del test di Coombs diretto, degli anticorpi antifattore piastrinico 4 (PF4), oltre a un debole incremento degli anticorpi antipiastrine, anticardiolipina (ACA: IgG 15,3 U/mL e IgM $17,13 \mathrm{U} / \mathrm{mL}$ ) e del fattore reumatoide. Interessante l'ecocardiografia di controllo che mostrava, oltre a minima falda di versamento pericardico residua, persistenza di piccola massa apicale ventricolare sinistra (fig. 1) del diametro di circa $10 \mathrm{~mm}$, flottante, con peduncolo adeso al setto, in assenza dei tipici segni di stratificazione e/o riassorbimento che ci saremmo aspettati da un trombo. Tale reperto strumentale, in associazione alla persistenza dei sintomi sistemici e dell'incremento degli indici di flogosi, ci induceva a ipotizzare che la piccola massa ventricolare sinistra fosse invece un mixoma in sede atipica, ipotesi che veniva confermata da TC e RM (figg. 2,3).

Durante la degenza la paziente presentava graduale miglioramento clinico e regressione della trombocitopenia; in considerazione del rischio elevato in paziente anziana con copatologie e delle esigue dimensioni della massa il cardiochirurgo sconsigliava un intervento di resezione, per cui manca una conferma istologica definitiva. Alla dimissione, avvenuta a distanza di 20 giorni, venivano proseguiti fondaparinux, insulina, levotiroxina, ramipril e si sospendeva gradualmente l'ASA. Un controllo ecografico agli arti inferiori a 3 mesi mostrava la completa ricanalizzazione venosa bilaterale, mentre al TTE la piccola "massa" apicale ventricolare sinistra era del tutto invariata per ecogenicità e dimensioni. Il fondaparinux veniva quindi embricato e poi sostituito con warfarin, che veniva proseguito per altri 4 mesi (range INR 2-3) e quindi sospeso.

La paziente è attualmente in ottime condizioni generali, nonostante lamenti saltuari episodi di flushing/sudorazione; gli indici di flogosi, la LAC-positività e il D-dimero sono rientrati nella norma. Persistono, d'altronde, positività degli anticorpi anti-PF4, degli anticorpi antipiastrine, del test di Coombs diretto ed elevati livelli plasmatici di IL-6, i cui valori

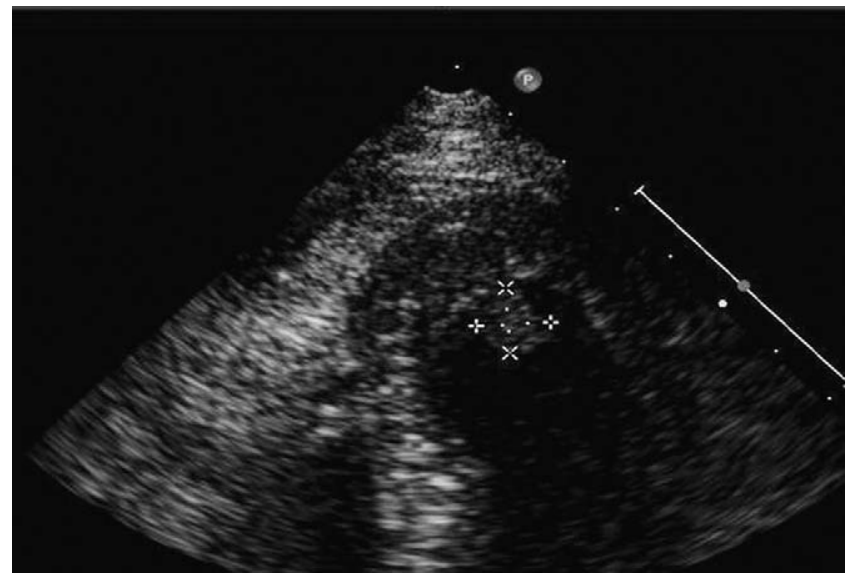

Figura 1 Immagine ecocardiografica della piccola massa in apice ventricolo sinistro.

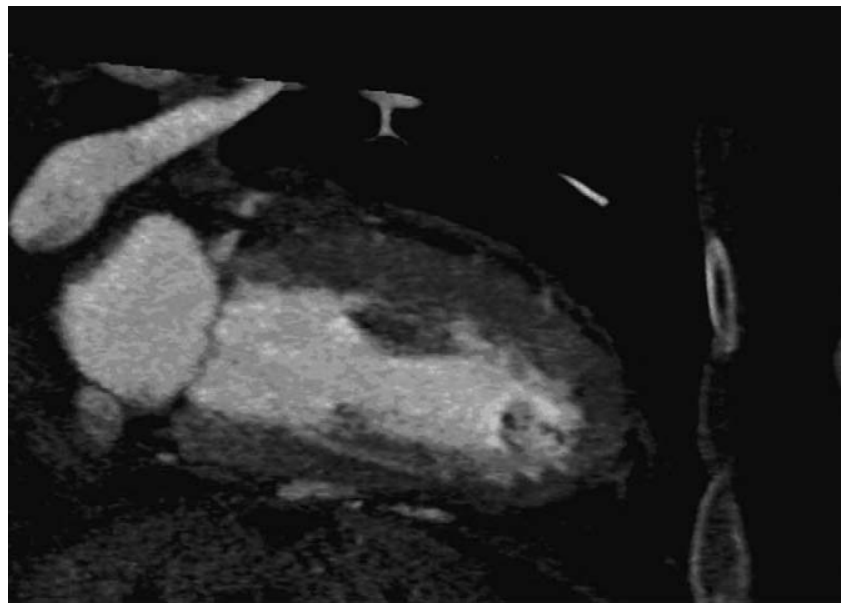

Figura 2 TC cardiaca del piccolo mixoma in apice ventricolo sinistro.

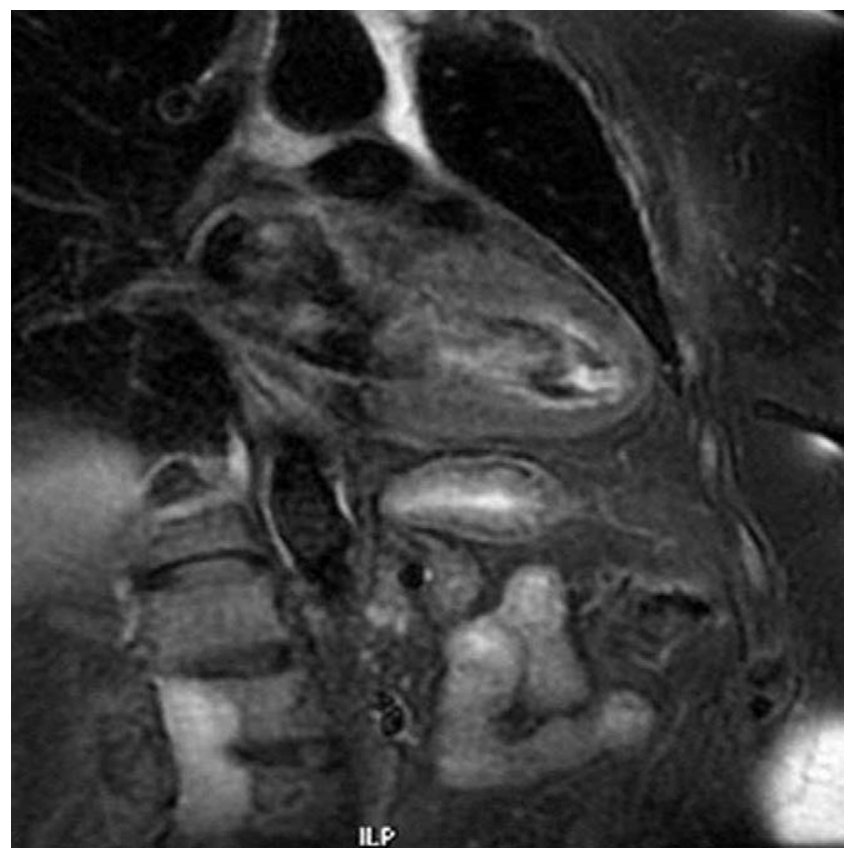

Figura 3 RM del mixoma in apice ventricolo sinistro.

(73,1 $\mathrm{pg} / \mathrm{mL}$, valori normali $0-4 \mathrm{pg} / \mathrm{mL})$ superano quelli riscontrati negli altri casi di mixoma descritti in letterarura [8,19-22]; essi sono invece paragonabili a quanto rilevato in patologie autoimmuni croniche quali l'arterite temporale [24], la polimialgia reumatica [25], la malattia di Takayasu [26] e l'artrite reumatoide [27].

\section{Discussione}

Dopo la prima descrizione di una neoformazione auricolare sinistra pubblicata su Lancet nel 1845 [28], la diagnosi di mixoma cardiaco rimase un puro reperto autoptico fino al 1952, anno in cui si documentò con angiocardiografia un tumore a livello atriale sinistro [29]. In seguito, l'avvento dapprima della metodica ecocardiografica $[30,31]$ e poi di TC 
e RM ha permesso diagnosi via via più raffinate di mixomi di dimensioni sempre più piccole nonché il trattamento, tutt'ora elettivamente chirurgico, sempre più precoce e in molti casi "salvavita". I mixomi sono infatti neoplasie benigne che tuttavia, in considerazione della sede e del potere emboligeno, possono avere gravi complicanze, fino al precoce e/o improvviso esito infausto $[1,32]$. La sintomatologia dei mixomi ha spettro molto ampio, estendendosi dal semplice riscontro casuale di tipo strumentale o autoptico alla morte improvvisa [1,10-16] (tab. 1).

Nonostante sia possibile un'accurata caratterizzazione tissutale con ecocardiografia [33], TC e RM, tutte in grado di precisare localizzazione, dimensioni, forma, mobilità, contorni, omogeneità o eterogeneità del segnale, vascolarizzazione, punto d'impianto ed eventuale presenza di aree emorragiche e/o necrotiche, il gold standard diagnostico è tuttora costituito dall'esame istologico [1,34]. La diagnosi differenziale (tab. 2) si pone con altre "neoformazioni" cardiache quali trombi $[35,36]$, rarissimi però in assenza di alterazioni predisponenti (come fibrillazione atriale, infarto miocardico acuto, ipocinesia globale o segmentarla, aneurismi del ventricolo sinistro), vegetazioni endocarditiche [37], altre rare neoplasie benigne e tumori maligni primitivi [38] o metastasi. Questi ultimi sono da 20 a 40 volte più frequenti e hanno localizzazione più spesso pericardica o miocardica rispetto a quella endocardica tipica dei mixomi [38,39]; come i mixomi, tuttavia, le neoplasia maligne cardiache e di altre sedi, come d'altronde le patologie autoimmuni o infettive, si associano con frequenza a sintomi sistemici, incremento degli indici di flogosi, alterazioni immunitarie e trombosi venose [1,10-12] (tab. 3).

Recenti segnalazioni $[6,19,21]$ mostrano un incremento di vari mediatori di flogosi e in particolare di IL-6 nei portatori di mixoma, a livelli tali da esser considerato un vero e proprio marker neoplastico secreto da parte delle cellule mesenchimali totipotenti da cui ha origine questa neoplasia. L'IL-6 è una citochina in grado di stimolare la produzione delle proteine della fase acuta e ha un livello appena dosabile nel plasma dei soggetti sani, mentre aumenta notevolmente in corso di patologie flogistiche, autoimmuni e aterosclerotiche quali artrite reumatoide, malattia di Castleman, infarto

Tabella 1 Mixoma cardiaco: sintomatologia "locale" correlata alla massa intracardiaca.

- Nessun sintomo: riscontro occasionale

- Sintomi ostruttivi: legati a dimensioni e localizzazione a livello delle camere cardiache Dispnea, episodi recidivanti di edema polmonare acuto o scompenso cardiaco destro, rottura di corde tendinee, ostruzione valvolare con sincope o morte improvvisa

- Aritmie

- Sintomi embolici: per frammentazione della massa neoplastica Nel mixoma cardiaco sinistro: prevalenti embolie cerebrali (attacchi ischemici transitori, ictus), ma anche sistemiche (embolie arteriose coronariche, mesenteriche, renali, periferiche) Nel mixoma cardiaco destro: embolia polmonare (monolaterale/bilaterale, gravità variabile)

- Embolie paradosse: in caso di forame ovale pervio con shunt - Morte improvvisa
Tabella 2 Mixoma cardiaco: diagnosi differenziale.

- Trombi intracavitari: quasi esclusivamente associati a condizioni predisponenti A livello locale: fibrillazione atriale (AS), infarto miocardico acuto, cardiopatia ipocinetico-dilatativa, aneurismi (VS), malformazioni cardiache (AS) A livello sistemico: trombofilia ereditaria (mutazioni del fattore II o V, deficit di antitrombina III, proteina C o S) o acquisita (neoplasie, sepsi, sindrome da anticorpi antifosfolipidi...)

- Altre neoplasie benigne: lipoma, fibroelastoma papillare, rabdomioma, neurofibroma

- Neoplasie maligne primitive: linfomi, rabdomiosarcomi

- Metastasi: linfomi, adenocarcinomi, sarcomi

- Vegetazioni endocarditiche: localizzate a livello di un apparato valvolare, più frequenti a livello mitralico e aortico

Legenda: $\mathrm{AS}$ = atrio sinistro; VS = ventricolo sinistro.

miocardico acuto, sepsi e psoriasi [21]. Sono riportati rarissimi casi di metastatizzazione e localizzazione plurifocale di mixoma [40-42], oltre a recidive dopo asportazione [22]; proprio in questi casi più "aggressivi", così come nei mixomi "complessi" o quelli in cui sono presenti sintomi e segni sistemici come febbre, sudorazione, dolore toracico, flushing, alterazioni immunitarie e flogistiche, si riscontrano le concentrazioni tissutali e/o plasmatiche più elevate di IL-6, il cui livello inoltre è riportato crollare dopo asportazione $[19,42]$ e risalire in caso di recidiva [19].

Il caso da noi descritto, pur in assenza di conferma istologica, presenta diverse caratteristiche cliniche e di laboratorio che rendono la diagnosi di mixoma la più verosimile $\mathrm{e}$ tendono a rendere meno probabile qualsiasi altro tipo di "massa" a livello del ventricolo sinistro:

- trombosi: esclusa da pattern ed evoluzione ecocardiografica, referto TC e RM e assenza di condizioni predisponenti;

- neoplasia maligna primitiva o metastatica: esclusa dalla favorevole evoluzione clinica in assenza di asportazione chirurgica o chemioterapia;

- vegetazione endocarditica: esclusa dalla localizzazione e dalla regressione dei sintomi sistemici in assenza di terapia antibiotica;

- vegetazione endocarditica atipica nel contesto di sindrome da anticorpi antifosfolipidi: esclusa da localizzazione, follow-up e successiva spontanea negativizzazione degli anticorpi antifosfolipidi.

Tabella 3 Mixoma cardiaco: segni e sintomi sistemici associati.

- Sintomi sistemici quali: febbre, calo ponderale, astenia, artralgie, sindrome di Raynaud

- Episodi ricorrenti di capogiri, flushing, sudorazione, ipotensione ortostatica, dispnea, dolore toracico

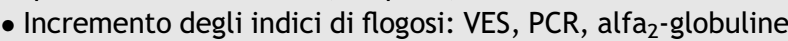

- Alterazioni immunitarie: positività del fattore reumatoide, anticorpi antinucleo, test di Coombs

- Anemia, leucocitosi, trombocitopenia

- Tromboembolismo venoso 
Inoltre, ci è sembrato di notevole interesse clinico per i seguenti motivi:

- sede atipica del mixoma con conseguente "misdiagnosi" iniziale;

- massiva attivazione sistemica dei fenomeni flogistici/ immunitari a fronte delle dimensioni irrilevanti della massa;

- comparsa di TVP prossimale bilaterale agli arti inferiori, verosimilmente in corso di HIT, ma in ogni caso nel contesto della "tempesta" autoimmune innescata dal mixoma, sia clinica (HIT, pericardite acuta, anemia emolitica autoimmune) sia di laboratorio (positività di anticorpi antipiastrine, anti-PF4, fattore reumatoide, LAC e test di Coombs);

- Livelli di IL-6 abnormemente elevati, con valori superiori a quanto riscontrato nei casi di mixoma finora descritti in letteratura e paragonabili a quelli riportati in corso di malattie autoimmuni quali arterite di Takayasu, arterite a cellule giganti, polimialgia reumatica (PMR) e artrite reumatoide [24-27];

- infine, remissione della TVP e dei segni/sintomi di tipo flogistico/autoimmune dopo trattamento con fondaparinux a fronte di livelli plasmatici di IL-6 ancora decisamente elevati.

Quest'ultimo dato, in considerazione delle esigue dimensioni della massa, ci induce a ipotizzare che esso rappresenti un marker non soltanto di mixoma ma anche di "attività" dello stesso, e possa indicare una possibile recidiva di "malattia sistemica", analogamente a quanto osservato in portatori di PMR con incremento isolato di IL-6 in corso di terapia steroidea [25]. Solo un attento follow-up ci permetterà di verificare se nel caso di mixoma da noi osservato un persistente incremento isolato di IL-6 si traduca sul piano clinico, oltre che nella persistenza dei sintomi sistemici "minori" quali flushing, capogiri, sudorazione, in un reale rischio di recidiva flogistico-trombotico-autoimmune.

\section{Conclusioni}

Il caso clinico presentato è fortemente suggestivo di mixoma cardiaco in sede atipica, complicato da fenomeni trombofilici e autoimmunitari, pur in assenza di conferma istologica, per la concordanza tra i test strumentali e l'immutabilità ecografica a un anno dalla diagnosi.

Peculiare del nostro caso è l'associazione con la TVP che, pur non essendo criterio diagnostico di mixoma cardiaco, deve essere considerata nel contesto di un processo flogistico-autoimmunitario sistemico di cui la chiave di volta sarebbe proprio la produzione di IL-6 da parte delle cellule mixomatose.

\section{Conflitto di interesse}

Gli autori dichiarano di essere esenti da conflitto di interessi.

\section{Bibliografia}

[1] Reynen K. Cardiac myxomas. N Engl J Med 1995;333(24):1610-7.

[2] MacGowan SW, Sidhu P, Aherne T, Luke D, Wood AE, Neligan MC, et al. Atrial myxoma: national incidence, diagnosis and surgical management. Ir J Med Sci 1993;162(6):223-6.
[3] Lie JT. The identity and histogenesis of cardiac myxomas. A controversy put to rest. Arch Pathol Lab Med 1989;113(7): 724-6.

[4] Meller J, Teichholz LE, Pichard AD, Matta R, Litwak R, Herman $M V$, et al. Left ventricular myxoma: echocardiographic diagnosis and review of the literature. Am J Med 1977;63(5):816-23.

[5] Keeling IM, Oberwalder P, Anelli-Monti M, Schuchlenz H, Demel $\mathrm{U}$, Tilz GP, et al. Cardiac myxomas: 24 years of experience in 49 patients. Eur J Cardiothorac Surg 2002;22(6):971-7.

[6] McCoskey EH, Mehta JB, Krishnan K, Roy TM. Right atrial myxoma with extracardiac manifestations. Chest 2000;118(2): 547-9.

[7] Chen J, He Y, Li Z, Han J, Gu X, Wang L, et al. Myxoma of the left ventricular outflow tract. J Ultrasound Med 2009;28(11): 1585-8.

[8] Mochizuki Y, Okamura Y, lida H, Mori H, Shimada K. Interleukin-6 and "complex" cardiac myxoma. Ann Thorac Surg 1998;66(3): 931-3.

[9] MCCarthy PM, Piehler JM, Schaff HV, Pluth JR, Orszulak TA, Vidaillet Jr HJ, et al. The significance of multiple, recurrent, and "complex" cardiac myxomas. J Thorac Cardiovasc Surg 1986;91(3):389-96.

[10] Pinede L, Duhaut P, Loire R. Clinical presentation of left atrial cardiac myxoma. A series of 112 consecutive cases. Medicine (Baltimore) 2001;80(3):159-72.

[11] Silverman J, Olwin JS, Graettinger JS. Cardiac myxomas with systemic embolization. Review of the literature and report of a case. Circulation 1962;26:99-103.

[12] Currey HL, Mathews JA, Robinson J. Right atrial myxoma mimicking a rheumatic disorder. Br Med J 1967;1(5539):547-8.

[13] Maisch B. Immunology of cardiac tumors. Thorac Cardiovasc Surg 1990;38(Suppl 2):157-63.

[14] Wiedermann CJ, Reinisch N, Fischer-Colbrie R, Vollmar AM, Herold M, Knapp E. Proinflammatory cytokines in cardiac myxomas. J Intern Med 1992;232(3):263-5.

[15] Kang N, Hughes CF. Massive pulmonary embolus complicating left atrial myxoma. J Thorac Cardiovasc Surg 2001;121(4): 814-5

[16] Labanti G, Urbinati S, Grepioni A, Poci MG, Pinelli G. Association of pulmonary embolism secondary to deep venous thrombosis and left atrial myxoma: case report and review of the literature. Ital Heart J Suppl 2003;4(7):598-601.

[17] Jourdan M, Bataille R, Seguin J, Zhang XG, Chaptal PA, Klein B. Constitutive production of interleukin- 6 and immunologic features in cardiac myxomas. Arthritis Rheum 1990;33(3): 398-402.

[18] Saji T, Yanagawa E, Matsuura H, Yamamoto S, Ishikita T, Matsuo $\mathrm{N}$, et al. Increased serum interleukin-6 in cardiac myxoma. Am Heart J 1991;122(2):579-80.

[19] Maze $Y$, Kajimoto M, Tenpaku H, Satou T. Left atrial myxoma with severe inflammatory response. Jpn J Thorac Cardiovasc Surg 2004;52(4):221-3.

[20] Esmon CT. Inflammation and thrombosis. J Thromb Haemost 2003;1(7):1343-8.

[21] Seino $Y$, Ikeda U, Shimada K. Increased expression of interleukin 6 mRNA in cardiac myxomas. Br Heart J 1993;69(6): $565-7$.

[22] Mendoza CE, Rosado MF, Bernal L. The role of interleukin-6 in cases of cardiac myxoma. Clinical features, immunologic abnormalities, and a possible role in recurrence. Tex Heart Inst $\mathrm{J}$ 2001;28(1):3-7.

[23] Frank RD, Schabbauer G, Holscher T, Sato Y, Tencati M, Pawlinski $\mathrm{R}$, et al. The synthetic pentasaccharide fondaparinux reduces coagulation, inflammation and neutrophil accumulation in kidney ischemia-reperfusion injury. J Thromb Haemost 2005;3(3): 531-40.

[24] Roche NE, Fulbright JW, Wagner AD, Hunder GG, Goronzy JJ, Weyand CM. Correlation of interleukin-6 production and disease 
activity in polymyalgia rheumatica and giant cell arteritis. Arthritis Rheum 1993;36(9):1286-94.

[25] Salvarani C, Cantini F, Niccoli L, Macchioni P, Consonni D, Bajocchi G, et al. Acute-phase reactants and the risk of relapse/recurrence in polymyalgia rheumatica: a prospective followup study. Arthritis Rheum 2005;53(1):33-8.

[26] Noris M, Daina E, Gamba S, Bonazzola S, Remuzzi G. Interleukin6 and RANTES in Takayasu arteritis: a guide for therapeutic decisions? Circulation 1999;100(1):55-60.

[27] Bajocchi GL, Pipitone N, Boiardi PL, Salvarani C. Artrite reumatoide: ruolo chiave dell'interleuchina- 6 nel network citochinico infiammatorio. Ital J Med 2008;2(4):40-6.

[28] King TW. On simple vascular growths in the left auricle of the heart. Lancet 1845;2:248-9.

[29] Goldberg HP, Glenn F, Dotter CT, Steinberg I. Myxoma of the left atrium; diagnosis made during life with operative and postmortem findings. Circulation 1952;6(5):762-7.

[30] Ha JW, Kang WC, Chung N, Chang BC, Rim SJ, Kwon JW, et al. Echocardiographic and morphologic characteristics of left atrial myxoma and their relation to systemic embolism. Am J Cardiol 1999;83(11):1579-82.

[31] Handke M, Schöchlin A, Schäfer DM, Beyersdorf F, Geibel A. Myxoma of the mitral valve: diagnosis by 2-dimensional and 3-dimensional echocardiography. J Am Soc Echocardiogr 1999;12(9):773-6.

[32] Pucci A, Gagliardotto P, Zanini C, Pansini S, di Summa M, Mollo F. Histopathologic and clinical characterization of cardiac myxoma: review of 53 cases from a single institution. Am Heart J 2000;140(1):134-8.

[33] Ohshima H, Kawashima E, Ogawa Y, Tobise K, Onodera S. Demonstration of the inner structure of a right atrial myxoma by transoesophageal echocardiography. Eur Heart J 1993;14(1): 132-4.

[34] Bjessmo S, Ivert T. Cardiac myxoma: 40 years' experience in 63 patients. Ann Thorac Surg 1997;63(3):697-700.

[35] Ghirarduzzi A, Galimberti D, Silingardi M, Cerioli GC, Parravicini $R$, Salvarani $C$, et al. Left atrial thrombosis in patients with antiphospholipid antibody syndrome and mesenchymal abnormal septum. Ital Heart J Suppl 2001;2(10):1111-6.

[36] DeGroat TS, Parameswaran R, Popper PM, Kotler MN. Left ventricular thrombi in association with normal left ventricular wall motion in patients with malignancy. Am J Cardiol 1985; 56(12):827-8.

[37] DePace NL, Soulen RL, Kotler MN, Mintz GS. Two dimensional echocardiographic detection of intraatrial masses. Am J Cardiol 1981;48(5):954-60.

[38] Feigenbaum H. Cardiac masses. In: Feigenbaum H, editor. Echocardiography. $5^{\text {th }}$ Ed., Philadelphia, PA: Lea \& Febiger; 1994. p. 589-629.

[39] Engberding R, Daniel WG, Erbel R, Kasper W, Lestuzzi C, Curtius $J M$, et al. Diagnosis of heart tumours by transoesophageal echocardiography: a multicentre study in 154 patients. European Cooperative Study Group. Eur Heart J 1993;14(9):1223-8.

[40] Shinfeld A, Katsumata T, Westaby S. Recurrent cardiac myxoma: seeding or multifocal disease? Ann Thorac Surg 1998;66(1): 285-8.

[41] Kanda T, Sakamaki T, Murata K. A cardiac myxoma with interleukin-6 production and cerebral metastasis. Int J Cardiol 1994;45(2):144-6.

[42] Takizawa T, Sumino H, Kanda T, Kobayashi I, Nagai R, Ichikawa S. An interleukin-6-producing cardiac myxoma associated with mediastinal lymphadenopathy. Cardiology 1999;92(4):275-7. 